\title{
Fatores associados ao óbito neonatal precoce em um estado do nordeste brasileiro
}

\author{
Factors associated with early neonatal debt in a northeast brazilian state \\ Factores asociados con la muerte neonatal temprana en um estado del noreste de Brasil
}

Recebido: 23/12/2020 | Revisado: 25/12/2020 | Aceito: 13/01/2021 | Publicado: 15/01/2021

\author{
Sônia Samara Fonseca de Morais \\ ORCID: https://orcid.org/0000-0002-0146-4611 \\ Universidade Estadual do Ceará, Brasil \\ E-mail: enfsoniasamara@hotmail.com \\ Cybelle Façanha Barreto Medeiros Linard \\ ORCID: https://orcid.org/0000-0001-7927-9320 \\ Universidade Estadual do Ceará, Brasil \\ Universidade Estácio do Ceará, Brasil \\ Centro Universitário Maurício de Nassau, Brasil \\ E-mail: cybellelinard@yahoo.com.br \\ Francisco Regis da Silva \\ ORCID: https://orcid.org/0000-0001-5470-2874 \\ Universidade Estadual do Ceará, Brasil \\ E-mail: regisfrs.silva@uece.br \\ José Auricélio Bernardo Cândido \\ ORCID: https://orcid.org/0000-0003-3327-8861 \\ Universidade Estadual do Ceará, Brasil \\ E-mail: jabcauricelio60@hotmail.com \\ Steffany Rocha da Silva \\ ORCID: https://orcid.org/0000-0003-3779-0977 \\ Universidade Federal do Ceará, Brasil \\ E-mail: psicologasteffany@gmail.com \\ Aline Ávila Vasconcelos \\ ORCID: https://orcid.org/0000-0002-3047-4093 \\ Universidade Estadual do Ceará \\ E-mail: alineavila@hotmail.com \\ Francisco José Maia Pinto \\ ORCID: https://orcid.org/0000-0002-4705-1513 \\ Universidade Estadual do Ceará, Brasil \\ Universidade Federal do Ceará, Brasil \\ E-mail: maia.pinto@yahoo.com.br
}

\begin{abstract}
Resumo
Objetivamos realizar uma análise dos fatores relacionados aos óbitos neonatais precoces ocorridos no Estado do Ceará. Estudo transversal, analítico, com uma amostra de 724 registros de óbitos neonatais precoces. Utilizamos como bases de informações: Sistema de Informação sobre Nascidos Vivos e Sistema de Informação sobre Mortalidade. Constatamos uma correlação entre o óbito neonatal precoce com as variáveis: anomalia; quantidade de consultas de pré-natal; Apgar no quinto minuto. A presença de anomalia nos recém-nascidos apresentou o dobro do risco quando relacionado ao óbito neonatal precoce. $\mathrm{O}$ mesmo foi observado referente a quantidade de consultas, indicando risco aumentado para ocorrer a mortalidade neonatal precoce de aproximadamente o dobro relacionado as gestantes que realizaram um quantitativo menor de que sete consultas pré natal. Outro parâmetro significativo foi o Apgar no quinto minuto, este mostrou aumento de seis vezes do risco de ocorrência de morte neonatal precoce. Evidenciou-se como mais importante para a origem dos óbitos dificuldades com relação ao pré-natal, parto e pós-parto. Todas essas questões refletem na atenção à saúde gestacional, bem como durante o crescimento e evolução relacionada ao recém-nascido.
\end{abstract}

Palavras-chave: Mortalidade neonatal precoce; Sistema de informação; Fatores de risco.

\begin{abstract}
We aimed to carry out an analysis of the factors related to early neonatal deaths that occurred in the State of Ceará. Cross-sectional, analytical study, with a sample of 724 records of early neonatal deaths. We use as bases of information: Information System on Live Birthsand Information System on Mortality. We found a correlation between early neonatal death and the variables: anomaly; number of prenatal consultations; Apgar in the fifth minute. The presence of anamaly in newborns presented twice the risk when related to early neonatal death. The same was observed with regard to the number of consultations, indicating an increased risk for early neonatal mortality of approximately twice that related to pregnant women who had less than seven prenatal consultations. Another significant parameter was Apgar in the fifth minute, which showed a six-fold increase in the risk of early neonatal death. It was found that difficulties in relation to prenatal, childbirth and posrpartum were more importante for the origino $f$ deaths. All of these issues are reflected in
\end{abstract}


the attention to gestacional health, as well as during the growth and Evolution related to the newborn.

Keywords: Early neonatal mortality; Information systems; Risk factors.

\section{Resumen}

Nuestro objetivo fue realizar un análisis de los factores relacionados con las muertes neonatales tempranas ocorridas en el estado de Ceará. Estudio transversal, analítico, con una muestra de 724 registros de muertes neonatales precoces. Usamos como bases de información: Sistema de Información sobre Mortalidad. Encontramos una correlación entre 1 amuerte neonatal precoz y las variables: anomalia; número de consultas prenatales; Apgar en el quinto minuto. La presencia de anomalia en los recién nacidos presentó el doble de riesgo cuando se relaciono con la muerte neonatal temprana. Lo mismo se observo con respecto al número de consultas, lo que indica un aumento del riesgo de mortalidade neonatal temprana de aproximadamente el doble que el relacionado con las embarazadas que tuvieron menos de siete consultas prenatales. Otro parâmetro significativo fue el Apgar en el quinto minuto, que mostro un aumento de seis veces en el riesgo de muerte neonatal temprana. Se encontro que las dificultades en relación cone 1 prenatal, el parto y el posparto fueron más importantes para el origen de las muertes. Todos estos temas se reflejan en la atención a la salud gestacional, así como durante el crecimiento y evolución relacionados con el recién nacido.

Palabras clave: Mortalidad neonatal temprana; Sistema de información; Factores de riesgo.

\section{Introdução}

A Taxa de Mortalidade Infantil (TMI) é um indicador social que está relacionada aos óbitos ocorridos em crianças, do momento do nascimento até completarem os primeiros 12 meses de vida. É um indicador relevante que dá visibilidade da prosperidade econômica de uma população, uma vez que demonstra o nível das ações relacionadas aos cuidados globais de saúde, como :prevenção de doenças, saneamento básico, educação de uma cidade, região ou de um país, entre outros (Ribeiro, 2018). Relacionada à TMI podem-se classificar três componentes: neonatal precoce que mensura os óbitos de neonatos de 0 a 6 dias; neonatal tardia, relacionado ao óbito infantil com 7 a 27 dias do nascimento, e pós-neonatal, que alcança os óbitos de 28 até 364 dias de vida completos, a cada mil nascidos vivos (Brasil, 2018a).

O Brasil, em 2015, ficou entre os 62 países que alcançou a meta de diminuição para dois terços da mortalidade infantil, proposta pela Organização das Nações Unidas (ONU), por meio dos Objetivos de Desenvolvimento do Milênio (ODM). Houve queda de $73 \%$, correspondente a uma redução de 61 mortes para 16 mortes a cada cem mil, em crianças com idade de até cinco anos (ONU, 2015). Essas taxas estão bem distantes de outros países desenvolvidos, como: Japão e Finlândia, que possuem dois óbitos para cada mil nascidos vivos; em patamares próximos da China, que possui TMI de 10,6; e bem abaixo de países da África Ocidental e Central, que estão próximas de uma TMI de 90 (UN, 2015). No Ceará, houve redução da TMI de 16,6 por mil, em 2013 para 15,07 por mil, em 2015. Essa redução reflete nos fatores que levam ao aumento da mortalidade em crianças, tais como: insuficiência na assistência e orientação às grávidas, deficiência da assistência hospitalar aos neonatos, ausência de saneamento básico e desnutrição. Esses fatores proporcionam risco à vida dos recém nascidos chegando a $71 \%$ dos óbitos em crianças menores de um ano (Cearà, 2015). Neste cenário, percebe-se a necessidade de se programar ações voltadas para o controle da mortalidade infantil, com foco no componente neonatal precoce no Brasil, visto que este representa cerca de $50 \%$ dos óbitos de crianças menores de um ano de idade (Brasil, 2013). Assim, torna-se indispensável a organização constante da agenda dos gestores de saúde em busca de se melhorar o acesso a assistência à saúde e estimular maiores investimentos em Unidade de Terapia Intensiva Neonatal que possam dar suporte à gravidez de alto risco. (Brasil, 2012).

Existem algumas estratégias que são empregadas no estabelecimento de um bom prognóstico em relação a mortalidade infantil neonatal, como: cálculo da idade gestacional, avaliação do peso, após o nascimento e escore de Apgar. Ambos estão associados à sobrevivência e, quando em combinação, podem determinar o bem-estar do recém-nascido, além de possíveis complicações associadas (Gomes, Et al., 2020). Evidências apontam que a elevada proporção de óbitos precoces em neonatos, nas primeiras 24 horas, tem relação direta com a qualidade da assistência prestada durante o pré-natal, o parto e o recém-nascido. Desta forma, os fatores que mais influenciam a mortalidade neonatal estão relacionados à prematuridade, ao baixo peso ao nascer e às complicações durante a gestação, parto e vitalidade da criança, após o nascimento (Jacinto, Aquino \& Mota, 2013). Algumas ferramentas são utilizadas para estabelecer prognóstico para o recém-nascido (RN), dentre elas: escore de Apgar, peso ao nascer e idade gestacional. Elas estão associadas à sobrevivência e, são uma medida do bem-estar do RN. Dessa forma, os valores de Apgar estipulam a vitalidade do RN, sendo valores maiores/iguais a sete, considerados bons. 
Estudos mostram a importância de se fazer investigações retrospectivas dos óbitos e estabelecer nexos causais a partir do perfil epidemiológico, determinando as causas mais frequentes e os fatores que podem intervir nesses óbitos (Jodas, et al., 2013). Assim, o foco na prevenção e o controle dos óbitos neonatais precoces, principalmente, nas primeiras 24 horas de vida, são ações importantes a serem investigadas com o objetivo de auxiliar gestores em saúde na tomada de decisão e planejamento das ações e/ou políticas públicas de saúde materno-infantil, que possam reduzir a mortalidade neonatal.

Objetivou-se analisar fatores relacionados aos óbitos neonatais precoces que ocorreram no Estado do Ceará.

\section{Metodologia}

Estudo epidemiológico transversal, com abordagem descritiva e analítica, realizado no Estado do Ceará, pertencente a uma região nordestina brasileira. Este estudo é parte integrante da Pesquisa de Mestrado Acadêmico intitulada "Perfil do Óbito Neonatal Precoce em um Estado do Nordeste Brasileiro" (Morais, 2015).

Os dados utilizados nesta pesquisa foram obtidos a partir do Departamento de Informática do Sistema Único de Saúde do Brasil (DATASUS), que é de livre acesso ao público. As informações colhidas foram retiradas em julho de 2015, com dados relativos a 2013, através do Sistema de Informação sobre Nascidos Vivos (SINASC) e o Sistema de Informação sobre Mortalidade (SIM). O SINASC registrou 24.876 nascidos vivos e o SIM contabilizou 1.726 registros de óbitos neonatais precoces (DATASUS, 2015).

A população foi composta pelo total de óbitos neonatais precoces ocorridos no Estado do Ceará, no período de 1 de janeiro a 31 de dezembro de 2013. A amostra inicial foi constituída a partir da extração e processamento dos dados com o auxílio do programa Tabwin, onde foi realizado o cruzamento dos bancos de dados SINASC e SIM. Como resultado do cruzamento foi obtido um quantitativo de 820 casos de óbitos neonatais. Como critérios de inclusão, foram considerados os registros de óbitos neonatais precoces, no qual, o local de parto e o endereço de residência da mãe fossem referentes ao estado do Ceará. Consideraram-se como critérios de exclusão, os óbitos neonatais com informações ausentes ou ignoradas (missing) nas variáveis designadas para análise, além dos registros de óbitos ocorridos em 2013, cujo nascimento fosse referente ao ano de 2012 . Para a amostra final, foram obtidos 724 registros de óbitos neonatais precoces.

Para coleta dos dados, utilizou-se instrumento semiestruturado, baseado nos dados contidos nas Declarações de Nascidos Vivos e Declarações de Óbito, onde foram retiradas informações para a escolha das variáveis maternas e dos recémnascidos. Os dados selecionados foram avaliados sob o aspecto da qualidade e confiabilidade, e em seguida, realizaram-se ajustes, retirando-se as variáveis que poderiam acarretar vieses na pesquisa. Considerou-se como desfecho, a ocorrência do óbito neonatal precoce dicotomizada (sim; não). Foram consideradas independentes, as seguintes variáveis categorizadas: óbito, gestação, parto e condições de nascimento.

A classificação obedeceu à seguinte ordem: 1. Características do óbito (idade do óbito: neonatal precoce - até 6 dias; neonatal tardio - 7 a 27 dias; pós-neonatal - 28 dias ou mais; óbito fora do hospital - sim ou não; causa básica do óbito - CID 10; município de ocorrência do óbito - todos); 2. Característica da gestação, do parto e condições de nascimento (tipo de parto: vaginal ou cesáreo; consultas de pré-natal (insuficientes <7) - sim ou não; gravidez múltipla - sim ou não; mãe adolescente - sim ou não; mãe com até 7 anos de estudo - sim ou não; mãe solteira - sim ou não); Apgar 5' (insatisfatório) - sim ou não; baixo peso ao nascer - sim ou não; pequeno para a idade gestacional - sim ou não; e presença de anomalia - sim ou não).

Os dados coletados foram analisados por meio do programa Stata®, versão 11.0. Inicialmente, fez-se a análise descritiva, através das frequências absolutas e percentuais. Na análise inferencial, utilizou-se a regressão de Poisson considerando-se no modelo não ajustado, o Qui-quadrado de Pearson, para avaliar a relação entre o desfecho com as variáveis que apresentaram valor descritivo inferior a 0,20. No modelo ajustado, apenas as variáveis que apresentaram nível de significância de $5 \%$ foram mantidas. Foram utilizadas ainda, as medidas da intensidade das associações, por meio da Razão de Prevalência (RP) com os intervalos de confiança 95\%, respectivos.

Esta pesquisa foi aprovada pelo Comitê de Ética em Pesquisa (CEP), de acordo com o parecer $n^{\circ}$ 939.670. Os aspectos éticos e legais que envolvem pesquisas com seres humanos foram respeitados e orientados a partir da Resolução 466/2012 do 


\section{Resultados}

Neste estudo evidenciaram-se 724 óbitos neonatais, no ano de 2013, no Estado do Ceará, sendo a maioria 676 (93\%) ocorrida em unidades de saúde hospitalares, com característica predominante a componente neonatal precoce 498 (68,8\%). As causas de mortes descritas nas declarações de óbito 260 (36\%) foi distribuída entre: septicemia bacteriana 114 (15,8\%); síndrome da angústia respiratória $58(8,0 \%)$; imaturidade extrema $46(6,4 \% \%)$ e asfixia ao nascer $42(5,8 \%)$. Foram mais prevalentes, em relação ao número óbitos registrados os municípios: Fortaleza 299 (41,5\%), Sobral $156(21,5 \%)$ e Juazeiro do Norte 4 (5,5\%) (Tabela 1).

Tabela 1 - Características dos óbitos Neonatais ocorridos no Estado do Ceará, 2013.

\begin{tabular}{lcc}
\hline Variáveis & $\mathbf{n = 7 2 4}$ & $\mathbf{\%}$ \\
\hline Idade do óbito (dias) & 498 & 68,8 \\
Neonatal precoce (até 6) & 131 & 18,1 \\
Neonatal tardio (7 a 27) & 95 & 13,1 \\
Pós-natal (28 ou mais) & & \\
Óbito fora do hospital & 48 & 6,6 \\
Sim & $\mathbf{6 7 6}$ & $\mathbf{9 3 , 4}$ \\
Não & & \\
Causas básicas do óbito & 46 & 6,4 \\
P072 - Imaturidade extrema & 42 & 5,8 \\
P219 - Asfixia ao nascer & 58 & 8,0 \\
P220 - Síndrome da angústia & & \\
respiratória & 114 & 15,8 \\
P369- Septicemia bacteriana & $\mathbf{4 6 4}$ & $\mathbf{6 4 , 0}$ \\
Outros ( $\geq 1$ e $<9$ casos) & & \\
Município de ocorrência do óbito & $\mathbf{2 9 9}$ & $\mathbf{4 1 , 5}$ \\
Fortaleza & 156 & 21,5 \\
Sobral & 40 & 5,5 \\
Juazeiro do Norte & 229 & 31,5 \\
Outros & & \\
\hline
\end{tabular}

N: tamanho da amostra; \%: frequência percentual. Fonte: SIM / SINASC.

Verificou-se, também, que mais da metade dos partos ocorridos foi: tipo vaginal 429 (59,3\%), com o número de consultas de pré-natal insuficientes 462 (63,8\%), não sendo mães adolescentes 619 (85,5\%), com escolaridade inferior a sete anos de estudo $465(64,2 \%)$, não solteiras 457 (64,0\%) e quase em sua totalidade não tinha histórico de gravidez múltipla 664 $(91,7 \%)$. No que diz respeito aos registros sobre as condições de nascimento das crianças, observou-se que a maioria indicou o Apgar no quinto minuto com grau insatisfatório, 399 (55,1\%); baixo peso ao nascer, 478 (66\%) e não eram pequenos para idade gestacional, 649 (89,6\%); sem registro de anomalias fetais 629 (86,9\%) (Tabela 2). 
Tabela 2 - Características da gestação, parto e das condições de nascimento ocorridos no Estado do Ceará, 2013.

\begin{tabular}{|c|c|c|}
\hline Variáveis & $n=724$ & $\%$ \\
\hline \multicolumn{3}{|l|}{ Gestação e parto } \\
\hline \multicolumn{3}{|l|}{ Tipo de parto } \\
\hline Vaginal & 429 & 59,2 \\
\hline Cesáreo & 295 & 40,8 \\
\hline \multicolumn{3}{|c|}{ Consultas de pré-natal } \\
\hline Insuficiente $(<$ sete $)$ & 462 & 63,8 \\
\hline Suficiente $(\geq$ sete $)$ & 262 & 36,2 \\
\hline \multicolumn{3}{|l|}{ Gravidez múltipla } \\
\hline Sim & 60 & 8,3 \\
\hline Não & 664 & 91,7 \\
\hline \multicolumn{3}{|l|}{ Mãe adolescente } \\
\hline $\operatorname{Sim}$ & 105 & 14,5 \\
\hline Não & 619 & 85,5 \\
\hline \multicolumn{3}{|c|}{$\begin{array}{l}\text { Escolaridade da mãe (até sete } \\
\text { anos) }\end{array}$} \\
\hline Sim & 259 & 35,8 \\
\hline Não & 465 & 64,2 \\
\hline \multicolumn{3}{|l|}{ Mãe solteira } \\
\hline Sim & 257 & 36,0 \\
\hline Não & 457 & 64,0 \\
\hline \multicolumn{3}{|c|}{ Condições de nascimento } \\
\hline \multicolumn{3}{|c|}{ Apgar 5' } \\
\hline Insatisfatório & 399 & 55,1 \\
\hline Satisfatório & 325 & 44,9 \\
\hline \multicolumn{3}{|l|}{ Baixo peso ao nascer } \\
\hline $\operatorname{Sim}(<2500 \mathrm{~g})$ & 478 & 66,0 \\
\hline Não $(\geq 2500 \mathrm{~g})$ & 246 & 34,0 \\
\hline \multicolumn{3}{|l|}{$\begin{array}{l}\text { Pequeno para idade } \\
\text { gestacional }\end{array}$} \\
\hline Sim & 75 & 10,4 \\
\hline Não & 649 & 89,6 \\
\hline \multicolumn{3}{|c|}{ Presença de anomalia } \\
\hline Sim & 95 & 13,1 \\
\hline Não & 629 & 86,9 \\
\hline
\end{tabular}

n: tamanho da amostra; \%: frequência relativa. Fonte: SIM / SINASC.

$\mathrm{Na}$ análise do óbito neonatal precoce com os possíveis fatores associados, observou-se significância estatística em relação à ocorrência do óbito: no hospital $(\mathrm{p}<0,004)$, tipo de parto $(\mathrm{p}<001)$, consultas de pré-natal (p<001), Apgar no quinto minuto insatisfatório ( $\mathrm{p}<001)$, baixo peso ao nascer com $(\mathrm{p}<001)$ e presença de anomalia $(\mathrm{p}<0,011)($ Tabela 3$)$. 
Tabela 3 - Fatores associados à mortalidade neonatal no Estado do Ceará, 2013.

\begin{tabular}{|c|c|c|c|c|c|c|c|}
\hline \multirow[t]{2}{*}{ Variáveis } & \multirow{2}{*}{$\begin{array}{c}\text { Total } \\
\text { n }\end{array}$} & \multicolumn{5}{|c|}{ Óbito neonatal precoce } & \multirow[b]{2}{*}{$\mathbf{p}$} \\
\hline & & $\%$ & Sim & $\%$ & Não & $\%$ & \\
\hline $\begin{array}{l}\text { Óbito fora do } \\
\text { hospital }\end{array}$ & & & & & & & $<0,004$ \\
\hline $\operatorname{Sim}$ & 48 & 6,6 & 24 & 50,0 & 24 & 50,0 & \\
\hline Não & 676 & 93,4 & 474 & 70,1 & 202 & 29,9 & \\
\hline Tipo de parto & & & & & & & $<0,001$ \\
\hline Vaginal & 429 & 59,3 & 317 & 73,9 & 112 & 26,1 & \\
\hline Cesárea & 295 & 40,8 & 181 & 61,4 & 114 & 38,6 & \\
\hline $\begin{array}{l}\text { Consultas de pré- } \\
\text { natal }\end{array}$ & & & & & & & $<0,001$ \\
\hline Insuficiente (< sete) & 462 & 63,8 & 341 & 73,8 & 121 & 26,2 & \\
\hline Suficientes ( $\geq$ sete) & 262 & 36,2 & 157 & 60,0 & 105 & 40,0 & \\
\hline Gravidez múltipla & & & & & & & $<0,169$ \\
\hline Sim & 60 & 8,3 & 46 & 77,0 & 14 & 23,0 & \\
\hline Não & 664 & 91,7 & 452 & 68,0 & 212 & 32,0 & \\
\hline Mãe adolescente & & & & & & & $<0,463$ \\
\hline $\operatorname{Sim}$ & 105 & 14,5 & 69 & 65,7 & 36 & 34,3 & \\
\hline Não & 619 & 85,5 & 429 & 69,3 & 190 & 30,7 & \\
\hline $\begin{array}{l}\text { Mães com até sete } \\
\text { anos de estudo }\end{array}$ & & & & & & & $<0,487$ \\
\hline Sim & 259 & 35,8 & 174 & 67 & 85 & 32,9 & \\
\hline Não & 465 & 64,2 & 324 & $\mathbf{7 0 , 0}$ & 141 & 30,0 & \\
\hline Mãe solteira & & & & & & & $<0,771$ \\
\hline Sim & 257 & 36,0 & 175 & 68,0 & 82 & 32,0 & \\
\hline Não & 457 & 64,0 & 316 & $\mathbf{7 0 , 0}$ & 257 & 30,0 & \\
\hline $\begin{array}{l}\text { Apgar 5' } \\
\text { insatisfatório }\end{array}$ & & & & & & & $<0,001$ \\
\hline Sim & 399 & 55,10 & 343 & 86,0 & 56 & 14,0 & \\
\hline Não & 325 & 44,90 & 155 & 47,6 & 170 & 52,4 & \\
\hline $\begin{array}{l}\text { Baixo peso ao } \\
\text { nascer }\end{array}$ & & & & & & & $<0,001$ \\
\hline Sim & 478 & 66,0 & 353 & 73,9 & 125 & 26,1 & \\
\hline Não & 246 & 34,0 & 145 & 59,0 & 101 & 41,0 & \\
\hline $\begin{array}{l}\text { Pequeno para } \\
\text { idade gestacional }\end{array}$ & & & & & & & $<0,246$ \\
\hline Sim & 75 & 10,4 & 56 & 74,6 & 19 & 25,4 & \\
\hline Não & 649 & 89,6 & 442 & 68,0 & 207 & 32,0 & \\
\hline $\begin{array}{l}\text { Presença de } \\
\text { anomalia }\end{array}$ & & & & & & & $<0,011$ \\
\hline Sim & 95 & 13,1 & 76 & 80,0 & 19 & 20,0 & \\
\hline Não & 629 & 86,9 & 422 & 67,0 & 207 & 33,0 & \\
\hline
\end{tabular}

n: tamanho da amostra; \%: frequência percentual; p: nível de significância do teste qui-quadrado de Pearson ou Exato de Fisher. Fonte: SIM / SINASC.

Após realização da análise não ajustada, das variáveis associativas em relação ao óbito neonatal precoce, observou-se que foram significativas, as variáveis: local do óbito ( $\mathrm{p}<0,005 ; \mathrm{RP}=0,43$; IC: 0,24-0,77); tipo de parto ( $\mathrm{p}<0,001 ; \mathrm{RP}=1,80$; IC: 1,30-2,45); número de consultas de pré-natal ( $\mathrm{p}<0,001$; $\mathrm{RP}=1,88$; IC: 1,35-2,60); Apgar no quinto minuto ( $\mathrm{p}<0,001 ; \mathrm{RP}=6,70$; IC: 4,70-9,60); baixo peso ao nascer (p<0,001; RP=1,95; IC:1,40-2,70) e anomalia (p<0,013; RP=1,95; IC:1,15-3,33). Ou seja, a relação do desfecho com as variáveis: tipo de parto, número de consultas de pré-natal, baixo peso ao nascer e anomalia apresentou risco aproximadamente de duas vezes de ocorrer óbito neonatal precoce, para cada uma delas. O Apgar apresentou aproximadamente sete vezes o risco de óbito neonatal precoce (Tabela 4). 
Tabela 4 - Modelo não ajustado: óbito neonatal precoce e variáveis associadas, Estado do Ceará, 2013.

\begin{tabular}{lccc}
\hline Variáveis & $\mathbf{R P}$ & $\mathbf{I C 9 5 \%}$ & $\mathbf{p}$ \\
\hline Local do óbito & $\mathbf{0 , 4 3}$ & 0,$24 ; 0,77$ & $<\mathbf{0 , 0 0 5}$ \\
Tipo de parto & $\mathbf{1 , 8 0}$ & 1,$30 ; 2,45$ & $<\mathbf{0 0 1}$ \\
Consultas de pré- & $\mathbf{1 , 8 8}$ & 1,$35 ; 2,60$ & $<\mathbf{0 , 0 0 1}$ \\
natal & & & \\
Gravidez múltipla & 1,55 & 0,$80 ; 2,85$ & 0,172 \\
Mãe adolescente & 0,85 & 0,$55 ; 1,30$ & 0,463 \\
Escolaridade da mãe & 0,90 & 0,$65 ; 1,25$ & 0,487 \\
Mãe solteira & 0,95 & 0,$70 ; 1,30$ & 0,771 \\
Apgar 5' & $\mathbf{6 , 7 0}$ & 4,$70 ; 9,60$ & $<\mathbf{0 , 0 0 1}$ \\
Baixo peso ao nascer & $\mathbf{1 , 9 5}$ & 1,$40 ; 2,70$ & $\mathbf{0 , 0 0 1}$ \\
Pequeno para idade & 1,40 & 0,$80 ; 2,40$ & 0,247 \\
gestacional & & & $\mathbf{0 , 0 1 3}$ \\
Presença de anomalia & $\mathbf{1 , 9 5}$ & 1,$15 ; 3,33$ & \\
\hline
\end{tabular}

n: tamanho da amostra; \%: frequência relativa; p: nível de significância do teste quiquadrado de Pearson ou Exato de Fisher; OR: odds ratio; IC: intervalo de confiança. Fonte: SIM / SINASC.

Após a regressão de Poisson ajustada, ao nível de significância 5\%, foram encontradas as seguintes variáveis associativas: anomalia ( $\mathrm{p}=0,024 ; \mathrm{RP}=1,96$; IC: 1,09-3,53); número de consultas de pré-natal ( $\mathrm{p}=0,018 ; \mathrm{RP}=1,57$; IC: 1,08-2,30); Apgar no quinto minuto ( $\mathrm{p}<0,001 ; \mathrm{RP}=5,9 ; \mathrm{IC}: 4,09-8,52)$. Assim, a existência da anomalia (para recém-nascidos) apresentou risco aproximado de duas vezes em relação ao óbito neonatal precoce. A variável número de consultas $(\mathrm{RP}=1,57)$ indicou risco aproximado de duas vezes de ocorrer a mortalidade neonatal precoce, dentre aquelas mães que realizaram menos de sete consultas. O Apgar no quinto minuto apresentourisco aproximado de seis vezes de ocorrer a morte neonatal precoce (Tabela 5).

Tabela 5 - Modelo final ajustado: regressão logística multivariada para fatores associados à mortalidade neonatal precoce, Estado do Ceará, 2013.

\begin{tabular}{lccc}
\hline Variáveis & RP ajustada & IC95\% & p \\
\hline Anomalia & $\mathbf{1 , 9 6}$ & 1,$09 ; 3,53$ & $<\mathbf{0 , 0 2 4}$ \\
Tipo de parto & 1,28 & 0,$89 ; 1,84$ & 0,174 \\
Consultas de pré- & $\mathbf{1 , 5 7}$ & 1,$08 ; 2,30$ & $<\mathbf{0 , 0 1 8}$ \\
natal & & & 0,069 \\
Baixo peso ao nascer & 1,42 & 0,$97 ; 2,08$ & $<\mathbf{0 , 0 0 1}$ \\
Apgar 5' & $\mathbf{5 , 9 0}$ & 4,$09 ; 8,52$ & $<$ \\
\hline
\end{tabular}

p: nível de significância do teste qui-quadrado de Pearson ou Exato de Fisher; OR: odds ratio; IC: intervalo de confiança. Fonte: SIM / SINASC.

\section{Discussão}

Nesta pesquisa evidenciou-se a ocorrência da maioria em hospitais nos municípios de Fortaleza, Sobral e Juazeiro do Norte. Este fato justifica-se por serem estes municípios os mais populosos do estado, onde estão localizados os hospitais de referência em alta complexidade, incluindo a assistência materno-infantil (Guilherme, 2017).

Nesta pesquisa observaram-se os óbitos neonatais relativos à mortalidade precoce. Este fato foi semelhante ao encontrado no Estado do Ceará, onde o óbito neonatal foi de $70 \%$ da mortalidade infantil e o componente mortalidade neonatal precoce respondeu por cerca de 50\% dos óbitos infantis (Rodrigues, 2018; Brasil, 2018).

Nesta pesquisa, a TMI inferior à encontrada em outras pesquisas, no Brasil, onde se observou que a taxa vinha diminuindo em todas as regiões, no período de 2000 a 2011: Região Norte de 16,9 para 10,4; Região Nordeste de 17,1 para 10,0; Região Sudeste, de 10,6 para 6,6; Região Sul de 8,5 para 5,8 e, Região Centro-Oeste de 11,3 para 8,1 por mil nascidos vivos (BRASIL, 2018B). Desta forma, observou-se que houve uma relação entre diminuição da taxa de óbitos neonatais no Ceará em comparação a região Nordeste. 
Percebeu-se que septicemia bacteriana foi a causa de maior prevalência dos óbitos neonatais precoces, bem superior ao encontrado na literatura. que correspondeu a $41 \%$ das ocorrências de óbitos na infância, seguido de outros componentes (França, et al., 2017). No entanto, outras pesquisas deram destaque para os transtornos respiratórios e cardiovasculares e somente depois relacionaram a septicemia como causa mais importante das mortes neonatais (Jacinto, Aquino \& Mota, 2013; Gaíva, Fujimori \& Sato, 2015). Presume-se, portanto, que as mortes precoces por septicemia se encontram em diminuição no Ceará.

É provável que as infecções neonatais em 2013 no Ceará, possam ter aumentado de corrente da superlotação das Unidades de Terapias Intensivas. Essas infecções têm alta incidência em neonatos e são de difícil controle devido à sua dependência com a qualidade dos serviços prestados durante a assistência à saúde no período pré-natal, perinatal e neonatal (Dias, 2013).

Em relação à Síndrome da Angústia Respiratória ou Síndrome do Desconforto Respiratório observou-se que o registro dos óbitos verificado neste estudo está diretamente relacionado ao amadurecimento das estruturas pulmonares e é responsável pela presença em $60 \%$ nos nascidos com menos de 30 semanas de gestacionais e em aproximadamente em $5 \%$ das crianças nascidas com menos de 37 semanas gestacionais (Nascimento, 2014).

A imaturidade extrema e a asfixia foram notificadas conforme pesquisa que evidenciou que estas causas interferem nos óbitos precoces, principalmente quando relacionadas ao baixo peso ao nascer (França \& Lansky, 2016; Hentges, et al., 2014).

A asfixia é responsável, ainda, a 25\% das mortes neonatais e cerca de $10 \%$ dos neonatos necessitam de intervenções para estabelecer a respiração nos primeiros minutos de vida. A cada três milhões de nascimentos por ano, aproximadamente 300.000 necessitam de assistência para estabilizar os movimentos respiratórios quando associada a hipóxia intrauterina (Daripa et al., 2013; Fernandes \& Rudek, 2015). O vômito é um agravo muito frequente entre os prematuros que desenvolvem algum agravo agudo, presente em mais de $50 \%$ dos prematuros (Rosa, et al., 2020).

Sobre as condições de nascimento, no mesmo ano, constatou-se que houve significância estatística para as variáveis o Apgar no quinto minuto e o baixo peso ao nascer, enquanto as variáveis relativas à idade da gestação e anomalias não apresentaram significância estatística (Tabela 2). Percebeu-se nos estudos que, quando o índice de Apgar possui inferioridade a sete, no quinto minuto, houve grau de sofrimento do neonato, relacionando disposições de assistência deficitárias, seja em nível de infraestrutura ou de recursos humanos das unidades de saúde, podendo contribuir para o óbito no primeiro dia de vida (Silva, et al., 2014; Daripa, et al., 2013).

A assistência imediata depois do parto diante de dificuldades obstétricas e indisponibilidade de UTI neonatal contribuiu para a sobrevivência do recém-nascido, enquanto o uso de medidas adequadas com recursos de infraestrutura e pessoal capacitado colabora para a diminuição em até 50\% dos óbitos neonatais (Kassar, 2013).

No presente estudo, observou-se, a partir do modelo ajustado, que o risco de óbito neonatal precoce no Ceará pode estar associado às variáveis: óbito ocorrido no hospital parto vaginal, consultas de pré-natal insuficiente, Apgar no quinto minuto insatisfatório, baixo peso ao nascer e presença de anomalia.

A partir dos dados obtidos, identificou-se semelhança, acerca do parto vaginal, com um estudo realizado em um município da região norte, cujo percentual de óbitos foi de 42,29\%. Contudo, a porcentagem de consultas de pré-natal não suficientes $(38,35 \%)$ foi relativamente menor que o percentual encontrado na presente pesquisa (Moreira, 2017).

Adicionalmente, na literatura é possível identificar semelhança aos dados encontrados, em que, o óbito no período neonatal está associado ao peso menor ao nascer, à anomalia e ao índice de Apgar abaixo de sete (Demitto, et al., 2017).

Os aspectos que têm associação ao período gestacional e parto levando em consideração ao número elevado de gestantes foram: quantidades insuficientes das consultas pré-natal escolaridade baixa, com inferioridade a sete anos de estudos, adultas e não solteiras. Quanto às características do parto, foi identificado que 59,2\% das mulheres com quantidade de consultas menor que sete encaminharam para parto vaginal, podendo ser associado a problemas gestacionais (Ferrari, Bertolozzi \& Dalmas, 2013).

Não foi verificada significância estatística para as variáveis: gravidez múltipla, gestação na adolescência, escolaridade da mãe, mãe solteira, ser considerado pequeno para idade gestacional. Também foi observado que esse resultado é diferente de outros já publicados, em que a idade e o nível de escolaridade da mãe foram variáveis que podem ser consideradas fatores de risco para o óbito neonatal. Pode-se indicar, assim, a importância da educação sobre saúde sexual e reprodutiva, atentando-se às 
necessidades de métodos contraceptivos e de planejamento familiar, e objetivando-se diminuir a incidência na adolescência (Morais, 2015). Foi possível identificar resultado semelhante com um estudo realizado nas cidades de Cuiabá e Londrina, cujo aspecto escolaridade da mãe não foi estatisticamente significante, diferenciando-se, também, ao que vem sendo mostrado na literatura (Ribeiro, et al., 2014).

Outrossim, foi possível identificar que o resultado deste estudo se assemelha aos obtidos em um estudo realizado em Pelotas e no Paraná, em que a associação possui significância estatística entre o índice de Apgar no quinto minuto e a morte intra-hospitalar de recém-nascidos (Santos, et al., 2014). É possível verificar semelhança de resultado com estudos realizados no Nordeste, como o peso baixo ao nascer e a maior magnitude de associação entre o índice de Apgar no quinto minuto e óbitos neonatais (Silva, et al., 2014; Silva \& Mathias, 2014).

Outros estudos publicados também retrataram resultados semelhantes quanto a relação de quantidade insuficientes de consultas, percebendo-se aumento em até 11,6 vezes do óbito neonatal. Quanto às anomalias congênitas, foi verificado estudos que mostram que entre mulheres com idade avançada há maior ocorrência. Quanto ao índice de Apgar menor que sete é possível identificar resultados semelhantes com esta pesquisa, indicando-se, assim, que essa variável também é considerada como fator de risco, sendo necessário o amparo imediato, visando diminuir as sequelas por falta de oxigenação no cérebro (Lansky, et al., 2014; Gaíva, Bittencourt \& Fujimori, et al., 2013; Kassar, et al., 2013).

Como limitação desse estudo, destaca-se as condições dos registros nos sistemas de informações, onde foi possível evidenciar a necessidade de melhorar a qualidade dos dados do SINASC e SIM. Desse modo, há necessidade de proporcionar treinamento, conscientização dos gestores e dos recursos humanos, no sentido de qualificar os dados dos sistemas de informações. Uma vez que estas devem ser preenchidas corretamente para que se possa conhecer melhor e implantar políticas de saúde com mais eficiência, identificando mais facilmente as necessidades de cada município, evitando atrasos nas medidas preventivas e desperdício de recursos. Portanto, é necessário também capacitar os profissionais que fazem acompanhamento de pré-natal e do parto na rede pública de saúde, por meio de especializações e residências que os habilitem a desenvolver a função com mais qualidade. Dar condições de trabalho estruturais: físicas e tecnológicas na atenção básica e nas maternidades públic as. Ampliar os comitês de investigação do óbito e trabalhar com os resultados obtidos como fonte de medidas preventivas a serem construídas e implantadas, a fim de minimizar os óbitos no estado do Ceará.

Acredita-se que esta pesquisa poderá contribuir com os profissionais de saúde que atuam no acompanhamento prénatal e no parto, ao suscitar informações acerca das características e fatores que se associam ao óbito neonatal precoce, ao passo que provoca reflexões acerca do planejamento de intervenções educativas, preventivas e assistenciais voltadas para a gestante e ao neonato. Espera-se que se possam trazer novos subsídios aos profissionais de saúde envolvidos no acompanhamento pré-natal e no parto no sentido de melhor direcionar o desenvolvimento dos recursos humanos que atuam diretamente com essas pacientes e neonatos.

Sugerimos continuidade nas pesquisas relacionadas ao acompanhamento pré-natal e ao parto visando uma maior contribuição aos recursos humanos que atuam diretamente com essas pacientes e neonatos.

\section{Conclusão}

Evidenciou-se que as principais causas dos óbitos têm como característica, problemas relacionados ao pré-natal, parto e pós-parto. Essa problemática reflete na qualidade da atenção à saúde gestacional, no crescimento e desenvolvimento do recémnascido.

\section{Referências}

Brasil. (2012). Ministério da Saúde. Conselho Nacional de Saúde. Diretrizes e normas regulamentadoras de pesquisa envolvendo seres humanos. Resolução n. 466, de 12 de dezembro de 2012. Brasília: Ministério da Saúde. http://bvsms.saude.gov.br/bvs/saudelegis/cns/2013/res0466_12_12_2012.html

Brasil. (2018a). Ministério da Saúde. Nascidos Vivos. Rede Interagencial de Informação para a Saúde RIPSA. http://tabnet.datasus.gov.br/cgi/tabcgi.exe?sinasc/cnv/nvCE.def 
Brasil. (2018b). Ministério da Saúde. Indicadores de Mortalidade. Rede Interagencial de Informações para a Saúde. http://tabnet.datasus.gov.br/cgi/idb2000/fqc01.htm

Brasil. (2018). Ministério da Saúde. Número de Óbitos na idade de 0 a 6 dias por 1.000 nascidos vivos Brasil, 2000-2011. Rede Interagencial de Informação para a Saúde RIPSA. http://tabnet.datasus.gov.br/cgi/idb2012/c0101b.htm

Brasil. (2012). Ministério da Saúde. Secretária de Atenção à Saúde. Departamento de Ações Programáticas Estratégicas. Atenção à Saúde do Recémnascido: guia para os profissionais de saúde/ Ministério da Saúde, Secretaria de Atenção à Saúde, Departamento de Ações Programáticas Estratégicas (2a ed.), Brasília: Ministério da Saúde http://bvsms.saude.gov.br/bvs/publicacoes/atencao_saude_recem_nascido_v1.pdf

Brasil. (2013). Ministério da Saúde. Secretaria de Vigilância em Saúde. Departamento de Análise da Situação em Saúde. Mortalidade infantil no Brasil: tendências, componentes e causas de morte no período de 2000 a 2010. In: Brasil. Secretaria de Ciência, Tecnologia e Insumos Estratégicos. Departamento de Ciência e Tecnologia. Síntese de evidências para políticas de saúde: reduzindo a mortalidade perinatal. Brasília: Ministério da Saúde/EVIPNet Brasil.http://bvsms.saude.gov.br/bvs/publicacoes/sintese_evidencias_reduzindo_mortalidade_infantil.pdf

Ceará. (2015). Secretaria de Saúde do Estado (SESA). Informe epidemiológico: mortalidade infantil. https://www.saude.ce.gov.br/wpcontent/uploads/sites/9/2018/06/informe_mortalidade_materna_junho_2015.pdf

Daripa, M. et al. (2013). Asfixia perinatal associada à mortalidade neonatal precoce: estudo populacional dos óbitos evitáveis. Rev. Paul Pediatria. 31(1), 3745. http://www.scielo.br/scielo.php?script=sci_arttext\&pid=S0103-05822013000100007

Datasus. (2015). Departamento de Informática do SUS. Sistema de Informação de Mortalidade. http://datasus.saude.gov.br/sistemas-e-aplicativos/eventos-v/simsistema-de-informacoes-de-mortalidade

Demitto, M. O. et al. (2017). Gestação de alto risco e fatores associados ao óbito neonatal [internet]. Revista da Escola de Enfermagem da USP, 51(3), e03208. https://www.scielo.br/scielo.php?pid=S00802342017000100409\&script=sci_abstract\&tlng=pt

Dias, V. M. O. (2013). Infecção relacionada à assistência à saúde precoce em unidade neonatal de alto risco [dissertação]. Fortaleza: Universidade Estadual do Ceará. http://www.repositorio.ufc.br/bitstream/riufc/4818/1/2013_dis_vmodias.pdf

Fernandes, M. C. \& Rudek, M. \& Souto, A.S. (2015). Recém-nascidos banhados em líquido amniótico meconial: atendimento em sala de parto e ocorrência de síndrome da aspiração meconial. Arq. Catarin Med. [internet], 44(4), 48-56. http://www.acm.org.br/acm/seer/index.php/arquivos/article/view/47

Ferrari, R. A. P. \& Bertolozzi, M.R. \& Dalmas, J. C. \& Girotto, E. (2013). Determining factors for neonatal mortality in a city in the Southern Region of Brazil. Revista da Escola de Enfermagem da http://www.scielo.br/scielo.php?pid=S008062342013000300531\&script=sci_arttext\&tlng=en

França, E. \& Lansky, S. (2016). Mortalidade Infantil Neonatal no Brasil: Situação, Tendências e Perspectivas [internet]. Anais do XVI Encontro Nacional de Estudos Populacionais -França. http://www.abep.org.br/publicacoes/index.php/anais/article/download/1763/1723

França, E.B. et al. (2017). Principais causas da mortalidade na infância no Brasil, em 1990 e 2015: estimativas do estudo de Carga Global de Doença. Rev. bras. epidemiol., [internet], 20(1), 46-60. http://www.scielo.br/scielo.php?script=sci_arttext\&pid=S1415-790X2017000500046\&lng=en\&nrm=iso

Gaíva, M. A. M. \& Bittencourt, R. M. \& Fujimori, E. (2013). Óbito neonatal precoce e tardio: perfil das mães e dos recém-nascidos. Rev. Gaúcha Enferm., [internet], 34(4), 91-97. http://www.scielo.br/scielo.php?script=sci_arttext\&pid=S1983-1447201300040001

Gaíva, M. A. M. \& Fujimori, E. \& Sato, A. P. S. (2015). Mortalidade neonatal: análise das causas evitáveis [Neonatal mortality: analysis of preventable causes]. Revista Enfermagem UERJ [internet], 23(2), 247-253. https://www.e-publicacoes.uerj.br/index.php/enfermagemuerj/rt/captureCite/5794/0

Gomes, F. E. S. et al. (2020). Impacto da implantação dos centros de parto normal na qualidade de assistência obstétrica. Research, Society and Development, 9(11), e3249119509. 10.33448/rsd-v9i11.9509. https://rsdjournal.org/index.php/rsd/article/view/9509.

Guilherme, M. (2017). Sistema Ceará Agora de Comunicação. Lista dos municípios do Ceará por população [internet]. http://www.cearaagora.com.br/site/listade-municipios-do-ceara-por-populacao/

Hentges, C. R. et al. (2014). Associação de sepse neonatal tardia com atraso do neurodesenvolvimento nos primeiros dois anos de vida de recém-nascidos prétermos de muito baixo peso. J. Pediatr. (Rio J.), 90(1), 50-57. http://www.scielo.br/scielo.php?pid=S0021-75572014000100050\&script=sci_abstract\&tlng=pt

Jacinto, E. \& Aquino, E. M. L. \& Mota, E. L. A. (2013). Mortalidade perinatal no município de Salvador, Bahia: evolução de 2000 a 2009. Rev. Saúde Pública [internet], 47(5), 846-853. http://www.scielo.br/scielo.php?script=sci_arttext\&pid=S0034-89102013000500846\&lng=en\&nrm=iso

Jodas, D. A. \& ScochiII, M. J. \& Vicente, J. B. \& Colucci, A.G. (2013). Análise dos óbitos evitáveis de menores de cinco anos no município de Maringá-PR. Esc. Anna Nery [internet], 17(2), 263-270. http://www.scielo.br/scielo.php?script=sci_arttext\&pid=S1414-81452013000200009\&lng=en\&nrm=iso

Kassar, S. B. \& Melo, A. M. C. \& Coutinho, S. B. \& Lima, M. C. \& Lira, P. I. C. (2013). Fatores de risco para mortalidade neonatal, com especial atenção aos fatores assistenciais relacionados com os cuidados durante o período pré-natal, parto e história reprodutiva materna. Jornal de Pediatria (Rio J) [internet], 89(3), 269-277. http://www.scielo.br/scielo.php?pid=S0021-75572013000300009\&script=sci_abstract\&tlng=pt

Lansky, S. et al. (2014). Pesquisa Nascer no Brasil: perfil da mortalidade neonatal e avaliação da assistência à gestante e ao recém-nascido. Cad Saude Publica [internet]. 39(1), S192-207. http://www.scielo.br/scielo.php?script=sci_arttext\&pid=S0102-311X2014001300024

Morais, S. S. F. (2015). Perfil do óbito neonatal precoce em um estado do nordeste brasileiro [dissertação]. Fortaleza: Universidade Estadual do Ceará. http://www.uece.br/cmasp/dmdocuments/S\%C3\%94NIA_SAMARA_FONSECA_DE_MORAIS.pdf

Moreira, K. F. A. \& Bicalho, B. O. \& Santos, L. C. S. \& Amaral, F. M. G. S. \& Orfão, N.H. \& Cunha, M. P. L. (2017). Perfil e evitabilidade de óbito neonatal em um município da amazônia legal [internet], 2(2), e48950. https://revistas.ufpr.br/cogitare/article/view/48950/pdf

Nascimento Júnior, F. J. M. \& Silva, J. V. F. \& Ferreira, A. L. C. \& Rodrigues, A. P. R. A. (2014). A síndrome do desconforto respiratório do recém-nascido: fisiopatologia e desafios assistenciais. Ciências Biológicas e da Saúde [Internet], 2(2), 189-198. https://periodicos.set.edu.br/index.php/fitsbiosaude/article/view/1836/1054

ONU - Organização das Nações Unidas. (2015). ONU: 16 MIL crianças morrem diariamente, revela novo relatório sobre mortalidade infantil 
[internet]. https://nacoesunidas.org/onu-16-mil-criancas-morrem-diariamente-revela-novo-relatorio-sobre-mortalidade-infantill/

Ribeiro A. (2018) "O que é mortalidade infantil?"; Brasil Escola [internet]. https://brasilescola.uol.com.br/o-que-e/geografia/o-que-e-mortalidadeinfantil.htm

Ribeiro, F. D. \& Ferrari, R. A. P. \& Sant'Anna, F. L. \& Dalmas, J. C. \& Girotto, E. (2014). Extremos de idade materna e mortalidade infantil: análise entre 2000 e 2009. Rev. Paul Pediatria [internet], 32(4), 381-388. http://www.scielo.br/scielo.php?pid=S0103-05822014000400381\&script=sci_arttext\&tlng=pt

Rodrigues, A.V. (2018). Ceará lança programa para reduzir mortes maternas e neonatais. Portal do Governo. Governo do Estado do Ceará [internet]. https://www.ceara.gov.br/2018/03/26/ceara-lanca-programa-para-reduzir-mortes-maternas-e-neonatais/

Rosa, N. P. da; Oliveira, D. C. de; Jantsch, L. B.; Neves, E. T. (2020). Agravos agudos de saúde de bebês prematuros moderados e tardios no período neonatal. Research, Society and Development, 9(7), e251974156. https://rsdjournal.org/index.php/rsd/article/view/4156.

Santos, H. G. \& Andrade, S. M. \& Silva, A. M. R. \& Mathias, T. A. F. \& Ferrari, L. L. \& Mesas, A. E. (2014). Mortes infantis evitáveis por intervenções do Sistema Único de Saúde: comparação de duas cortes de nascimentos. Ciênc. Saúde Coletiva [Internet], 19(03):907-16. https://www.redalyc.org/pdf/630/63030163025.pdf

Silva, A. L. \& Mathias, T. A. F. (2014). Fatores de risco independentes associados a óbitos infantis. Acta Paul Enferm., [internet], 27(1), 48-55. http://www.scielo.br/scielo.php?pid=S0103-21002014000100011\&script=sci_arttext\&tlng=pt

Silva, C. F. \& Leite, A. J. M. \& Almeida, N. M. G. S. \& Leon, A. C. M. P. \& Olofin, I. \& Perinatal, R. N. N. S. (2014). Fatores associados ao óbito neonatal em recém-nascidos de alto risco. Cad. Saúde Pública [internet], 30(2), 355-368. http://www.scielo.br/scielo.php?pid=S0102311 X2014000200355\&script=sci_abstract\&tlng=pt

UN. (2015). United Nations, Department of Economic and Social Affairs, Population Division (2015). World Population Prospects: The 2015

Revision, DVD Edition [internet]. https://esa.un.org/unpd/wpp/publications/files/key_findings_wpp_2015.pdf 